\title{
'The word of war' : vers une rhétorique de la guerre
}

\section{Margaret Jones-Davies}

\section{(2) OpenEdition}

\section{Journals}

Édition électronique

URL : http://journals.openedition.org/shakespeare/1208

DOI : 10.4000/shakespeare.1208

ISSN : 2271-6424

Éditeur

Société Française Shakespeare

Édition imprimée

Date de publication : 1 novembre 1990

Pagination : 11-24

Référence électronique

Margaret Jones-Davies, "'The word of war' : vers une rhétorique de la guerre », Actes des congrès de la Société française Shakespeare [En ligne], 8 | 1990, mis en ligne le 01 janvier 2007, consulté le 03 mai 2019. URL : http://journals.openedition.org/shakespeare/1208; DOI : 10.4000/shakespeare.1208 


\section{SHAKESPEARE \\ ET LA GUERRE}

Ouvrage publié avec le concours de la Commission

des Communautés Européennes 
Illustration de la couverture : DERRICKE'S Image of Ireland, 1586 


\section{«The word of war»: vers une rhétorique de la guerre}

La personnification de la rhétorique dans le livre $\mathrm{V}$ du Mariage de Philologie et de Mercure de Martianus Capella ${ }^{1}$ nous fait penser à ces représentations de la Renaissance où Vénus se déguise en Mars. Comme Vénus, elle séduit et comme Mars elle terrifie. Sous sa couronne royale elle porte un casque. Elle est armée. Ses vêtements sont recouverts de bijoux, certes, mais lorsqu'elle marche, ceux-ci résonnent contre son armure. Elle a le pouvoir d'une reine sur toute chose, peut changer la politique des Etats et des armées. C'est qu'à cette époque, le cinquième siècle, la rhétorique joue encore un rôle civil, elle prend part aux affaires de l'Etat. Dans ses Etymologies, Isidore de Séville la définit comme «bene dicendi scientia in civilibus quaestionibus $»^{2}$. Née dans les procès siciliens des propriétaires spoliés de leurs terres sous le règne des tyrans, elle est un instrument de la politique. Dans sa Rhétorique, Aristote énumère les cinq sujets les plus importants sur lesquels délibèrent tous les hommes: «les revenus, la guerre et la paix, la protection du territoire, l'importation et l'exportation, la législation $»^{3}$.

La guerre est un objet de la rhétorique. L'une des fonctions de la rhétorique serait donc de déterminer l'opportunité de la guerre. Mais si le discours est le fondement de la légitimité de la guerre, si, en d'autres termes, la cause de la guerre n'est qu'un mot, «a word of war » (Ant, II.ii.44), comme c'est le cas dans la guerre entre César et Antoine, alors on peut craindre l'emprise des sophistes dans la Cité. C'est ce que craignait Platon, qui avec précaution opposait deux types de rhétorique, celle fondée sur le vraisemblable et celle fondée sur la vérité. 
. Shakespeare aussi oppose comme Platon deux sortes de rhétorique à l'œuvre dans les affaires de la guerre. Dans Henry $V$, il les nomme de deux manières différentes: «the discourse of war» et «the phrase of war». La première est positive:

List his discourse of war and you shall hear

A fearful battle rendered you in music ( $H 5$, I.i.43-4)

Cette rhétorique est puissante car elle fait l'alliance des contraires comme les gardiens de la cité platonicienne qui parviennent à maintenir la paix parce qu'ils «ont un naturel à la fois doux et irascible ${ }^{4}$. On pense à Theseus, le guerrier devenu paisible de $A$ Midsummer Night's Dream qui parle en oxymores et dont les chiens font résonner dans les bois de Crète «so musical a discord» (MND, IV.i.117), et qui se demande «how shall we find the concord of this discord? «(MND, V.i.60). Ce discours de la guerre est harmonieux comme de la musique. Il s'inspire de la conception platonicienne, selon laquelle la guerre née du péché ${ }^{5}$ 'est justifiable qu'en unissant les contraires pour aboutir à la paix ${ }^{6}$, idée que reprendra St Augustin dans La Cité de Dieu?.

À cette première rhétorique de la guerre s'oppose une autre rhétorique, négative, identifiable par l'expression «the phrase of war» (H5, III.vi.74). Cette opposition marque la volonté d'une présentation dialectique de la guerre. C'est un discours fanfaronnant, appris par cœur, excluant toute forme de sagesse et glorifiant des guerres mercenaires. Il évoque le discours du soldat dans le dialogue d'Erasme entre le soldat et le chartreux ${ }^{8}$. Cette rhétorique rappelle l'attitude naturellement belliqueuse de ceux qui, selon Platon, dans Le Politique, «inclinent plutôt vers la force et poussent leur pays à quelque guerre par suite de leur passion trop violente pour ce genre de vie ${ }^{9}$. Le texte shakespearien montre la facilité avec laquelle on peut glisser d'un discours à l'autre: Henry luimême se laisse parfois emporter par une certaine violence 
peu musicale qui le fait s'arrêter et demander pardon à Dieu :

\section{Yet forgive me God}

That I do brag thus (H5, III.vi.149)

se faisant l'écho d'un autre guerrier qui, hésitant entre les deux rhétoriques de la guerre, celle qui cherche à éviter la guerre, et celle qui exprime la passion belliqueuse, s'accusera aussi de fanfaronnade mais y cèdera finalement. Il s'agit d'Hector. "You wisest Grecians, pardon me this brag» (Tro, IV.v.256), dira-t-il. Mais Ajax devra pourtant lui rappeler que le conseil de guerre ne peut en aucun cas justifier sa colère «privée» contre Achille:

The general state I fear

Can scarce entreat you to be odd with him (Tro, IV.v.263)

Cette «phrase of war» porte en elle toutes les mauvaises raisons de faire la guerre. A la différence du «discourse of war», «the phrase of war » n'est autre que ce que Lepidus appelle dans Antony and Cleopatra, "the speech of passion» (Ant, II.ii.12). Ceux qui en sont les victimes sont ceux pour qui la guerre et la paix sont des valeurs indifférentes. C'est le cas d'Antoine qui, par simple galanterie, informe Cléopâtre que ses désirs déterminent la guerre ou la paix,

making peace of war

As thou affects (Ant, I.iii.70-1)

tels les chiens de la populace dont parle Caius Martius et qui n'aiment ni la guerre ni la paix (Cor, I.i.166-7).

Ce relativisme moral concernant la politique de la guerre est bien décrit dans le passage déjà cité du Politique. Mais le danger ne vient pas seulement de ceux qui 
font la guerre pour le plaisir. Il vient aussi de ceux qui «poussent l'amour de la modération au-delà des bornes raisonnables et deviennent inconsciemment incapables de faire la guerre ${ }^{10}$. La structure contrastive de Troilus and Cressida est fondée sur l'opposition platonicienne entre ces deux cas extrêmes: Hector justifie la guerre par sa passion et demande qu'Hélène reste à Troie. Achille justifie sa passivité par sa passion pour l'une des filles de Priam, qui lui rend la paix aimable. Ce parallélisme presque comique est le résultat d'une sophistique du désir qui aboutira à la conclusion que l'on sait et qui s'oppose à une rhétorique fondée sur une volonté rationnelle de déterminer l'opportunité de l'action guerrière ou non.

Cette opposition entre ces deux rhétoriques semblerait accréditer l'idée que l'œuvre de Shakespeare est une illustration des théories platoniciennes sur la guerre. Mais parfois, au-delà de ce qu'on a pu appeler le pacifisme platonicien ${ }^{11}$, on sent l'influence belliciste de certains théoriciens médiévaux, qui, se fondant sur la tradition aristotélicienne de la guerre dans La Politique ${ }^{12}$, la considèrent comme naturelle au même titre que la chasse et donc nécessaire à la survie. Or, comme le dit Koyré «c'est Aristote qui fit l'éducation politique de l'Europe $»^{13}$. Dans son article sur «Quelques aspects de la pensée de la guerre au moyen-âge», J. Quillet étudie les arguments suspects de ces grands penseurs qui finissaient par enlever à la guerre son aspect maléfique en lui conférant un caractère fatal, inhérent à la nature humaine ${ }^{14}$.

Au-delà des différences idéologiques sur la nature de la guerre, ce qui préoccupe Shakespeare est le lien entre le langage et la guerre, et, plus précisément, la possibilité de légitimer la guerre dans le discours. C'est pourquoi la thématique de la guerre s'intègre dans une discussion plus vaste sur les limites de la rhétorique, sur ce que Platon craignait tant: les détournements sophistiques dont $\mathbf{R}$. Laufer analyse les conséquences dans son article «Rhétorique et Politique ${ }^{15}$. Ainsi divers points de vue contradictoires s'affrontent dans ses pièces, des tragédies aux histoires, aux comédies, comme si dans le grand labora- 
toire de son œuvre il cherchait à exposer les divers cas de figure de la terrifiante dialectique des mots et de la force.

D'abord la force ne doit jamais servir à justifier les mots: ce ne peut être qu'une sorcière comme Jeanne la Pucelle qui cherche par la force à prouver la vérité de ses mots (IH6, I.ii.96). Volumnia réprouve Coriolan qui voudrait retrouver son nom perdu en mettant Rome à feu et à sang (Cor, V.i.11-5). Richard II voudrait armer son nom ( $R 2$, III.ii.85-7), et c'est également un signe de faiblesse lorsque Richard III niera la force du mot conscience:

Conscience is but a word that cowards use Our strong arms be our conscience, swords our law (V.iii.310-1)

Par contre les mots ne doivent pas entraver l'usage de la force. Ce sont les lâches comme Falstaff «sweet creature of bombast» (IH4, II.iv.320), ou Pistol, qui ont «a killing tongue and a quiet sword» (H5, III.ii.34). Quant au «bald unjointed chat» du «popinjay» de I Henry $I V$, il peut déstabiliser le délicat équilibre lorsqu'il se mêle de parler

so like a waiting-gentlewoman

Of guns and drums and wounds (I.iii.54-5).

Un soldat ne parle pas. Henry V est satisfait que Kate ne parle pas anglais. Cela lui évitera de faire des prouesses d'éloquence insupportables pour un soldat (H5, V.ii. 120$60)$.

Et pourtant ce sont les mots, les mots dangereux dont on parle à l'acte V de Julius Caesar, ceux qui s'assimilent si bien aux coups (V, i, 27-37) qui doivent fonder l'acte de guerre. «We shall talk before we fight», diront Pompée et César (Ant, II.vi.2).

Pour légitimer l'énorme pouvoir de cette rhétorique, en analyser les limites, Shakespeare réintroduit les grands concepts de la philosophie nominaliste, le nom, la substance, l'universel, l'individu. Qu'est donc le mot de la 
guerre qui enverra à la mort des milliers d'hommes? La guerre qui en naîtra ne sera-t-elle pas une guerre d'ombres? A quelle condition le langage pourra-t-il assumer cette responsabilité? Quelle rhétorique permettra finalement au mot de se substituer à la force pour prouver, le temps d'une comédie, la non fatalité de la guerre? Que nous dit ce «word of war» qui, dans Antony and Cleopatra, recouvre le mot «cause»?

C'est le synonyme de «motive» (Ant, II.ii.95), luimême équivalent de cette cause qui, à côté d' «auctoritas, d'affectus, d'intentio, de conditio, de meritum ${ }^{16}$, fondait la guerre juste. Mais Shakespeare ici remplace «cause» par «word» comme s'il voulait en marquer le caractère arbitraire. Plus tard, «the word of war» sera qualifié de «magical» (Ant, III.i.31). Les actes de guerre, dans de telles conditions sont gouvernés par de ces mots qui semblent planer au-dessus de la tête des combattants comme les «paroles gelées» du livre lvi du Quart Livre et qui, en dégelant, rendaient des sons de tambours. ${ }^{17} \mathrm{La}$ cause des guerres entre Pompée, César et Antoine n'étant qu'un mot, elle n'a aucune substance, et la guerre est une guerre d'ombres. Ventidius et Silius le savent bien qui qualifient de magique ce mot qui ne se réfère qu'à des généraux absents:

I'll humbly signify what in his name,

That magical word of war, we have effected. (Ant, III.i. 30-1)

La seule réalité de la guerre ne serait-elle que les soldats? En tout cas se pose la question de la réalité de ce mot de la guerre qui agit en l'absence de tout substrat corporel. Par deux fois dans la pièce, il est question de l'absence des généraux sur le champ de bataille: ici, lorsque Ventidius rapporte que lui et Silius sont les vrais responsables de la victoire et lorsqu'Antoine se demande s'il était possible que César fût effectivement présent en personne à Toryne (III.vii.55). C'est comme si le discours voulait, en retirant les généraux du théâtre des opéra- 
tions, démontrer le pouvoir du mot seul. Ce mot, c'est Antoine, c'est lui la cause de la guerre. A l'acte IV, il se dit être un nuage (IV.ii.27) comme si en refusant de se donner comme référant au nom il en assumait l'immatérialité, et son nom, tel un mot magique, acquiert une matérialité si bien qu'il finit par se casser comme un objet:

Then in the midst a tearing groan did break The name of Antony. (Ant, IV.xiv.31-2)

La guerre devient dès lors sorcellerie, comme celle qu'engage la Pucelle. Elle gagne Orléans en utilisant ainsi qu'Hannibal les stratagèmes de la peur $($ IH6, I, v, 20). De même Richard III, qui accorde plus de réalité aux ombres qu'à «the substance of ten thousand soldiers/Armed in proof and led by shallow Richmond» $(R 3$, V.iii.217-220). Les corps substantiels des soldats ne comptent pour rien dans ces guerres d'ombre dont la cause n'est, au dire de Hamlet, que «a fantasy and trick of fame» (Ham, IV.iv.61). La cause de la guerre n'est qu'un mot, même s'il s'agit en fait d'un bien réel lopin de terre:

We go to gain a little patch of ground

That hath in it no profit but the name (Ham, IV.iv.18-9)

Ce qui semblait chez Aristote donner une réalité ontologique à la guerre, la propriété vitale à la survie décrite dạns sa Politique ${ }^{18}$, se réduit ici à une abstraction à laquelle on a magiquement, donc artificiellement, donné une réalité.

L'étude de tels exemples pourrait laisser croire qu'une description nominaliste du nom posé comme «cause» de la guerre interdise la justification de toute guerre, enlève à cette cause son substrat ontologique, la rende à tout jamais suspecte. Les paroles de Hamlet résumeraient cette attitude. Mais le texte shakespearien fournit une contre interprétation du nom de la guerre. Aux Français qui mettaient leur espoir dans la parole d'une sorcière, Talbot oppose le nom de Dieu: 
Well let them practise and converse with spirits God is our fortress, in whose conquering name Let us resolve to scale their flinty bulwarks (IH6 II.i.25).

Alors un simple soldat anonyme peut conférer à ce nom toute la réalité d'une arme:

The cry of Talbot serves me for a sword For I have loaden me with many spoils Using no other weapon but his name (IH6, II.i.7881)

Le nom du roi ne suffit pas à fonder une cause juste. Richard III pouvait dire «the king's name is a tower of strength» $(R 3$, V.iii.12), mais ce cri de guerre n'était qu'une fanfaronnade à côté du cri de Richmond qui ordonnait à ses soldats d'avancer «in God's name» $(R 3$, V.ii.22). Henry $\mathrm{V}$ a gagné la guerre car selon Winchester, «the battles of the Lord of Hosts he fought» (IH6,I.i.31). Dieu seul peut conférer une réalité au nom de la guerre. Ainsi on peut dire que ce recours à la métaphysique justifie une ontologie de la guerre chez Shakespeare qui se fonde sur une conception chrétienne et médiévale de la guerre. Les limites de la rhétorique sont posées. On entre dans le domaine de la métaphysique ${ }^{19}$. La problématique ontologique est poussée très loin dans l'analyse de Talbot. La Comtesse d'Auvergne veut tester la réalité du nom de Talbot. Fascinée par ce qu'elle croit n'être qu'un mirage elle veut en venir à bout en emprisonnant le corps de Talbot et en le couvrant de mépris. Il n'est qu'une «writhled shrimp» (IH6, II.iii.22). Elle veut déduire de l'apparente insubstantialité du corps de Talbot l'insubstantialité de la cause de la guerre. Mais le véritable corps de Talbot est son armée à laquelle Dieu confère son pouvoir. Comparons avec les soldats d'Antoine qui ont prêté leur corps à son nom vide :

Not as you serv'd the cause, but as't had been 
Each man's like mine. You have shown all Hectors (Ant, IV.viii.5-7)

La cause n'a pas de réalité en dehors de la personne d'Antoine. Elle n'est donc qu'un mot. L'acte guerrier est un acte de bravoure désespéré, l'allusion à Hector, «Bellona's bridegroom» s'il en est, le dit bien.

La guerre shakespearienne est décrite en termes d'ombres et de substance, le mot s'oppose à l'être, se gorge de la substance de cet être pour le réduire en ombre. La description nominaliste de la guerre se corrige d'une référence ontologique à Dieu qui donne une place au-delà du «pacifisme» platonicien à l'idéologie belliciste du Moyen-Age. Doit-on s'arrêter à l'hypothèse que, dans l'œuvre, l'argumentation nominaliste ne servirait que de repoussoir à une ontologie de la guerre qui paraderait dans les pièces historiques où se lirait le mode d'emploi de la guerre juste? Concession à l'histoire belliqueuse de l'Angleterre dont les Tudors maintiennent le mythe? Ce n'est heureusement pas si simple car au cœur de la pièce historique qui semble le plus justifier une certaine idée glorieuse de la guerre, Henry $V$, on voit apparaître un autre aspect de la problématique nominaliste: c'est le combat de l'Universel et de l'individuel, véritable combat de Jacob et de l'Ange qui nous permettra de réconcilier les pièces «médiévales» où la métaphysique semble venir à bout de la rhétorique avec les pièces où triomphe une rhétorique dangereuse pour ce type d'idéologie.

La guerre de Henry $V$ réunit les conditions de moralité requises par la tradition platonicienne et par saint Thomas $^{20}$. L'autorité du conseil a délibéré avant d'engager le combat, la cause est dite juste (IV.i.124), «well-hallowed» (I.ii.294). L'intentio est pacifique, comme celle de Malcolm. La guerre est décrite négativement. Elle a d'horribles mâchoires (II.iv.93). Elle est «impious/ Arrayed in flames like to the prince of fiends" (III.iii.15-6). C'est pourtant dans cette pièce que la rhétorique se fait omniprésente, comme si elle ne voulait pas laisser le dernier mot à l'ontologie, comme si elle voulait 
sans cesse rappeler que la guerre doit avant tout se légitimer dans le discours. Elle se manifeste dans tous ses genres: épidictique dans le prologue par exemple, où après avoir demandé à la muse de feu de l'aider à trouver matière à l'inventio, le chœur nous présente la guerre comme un spectacle (Prologue 5-7); juridique dans le discours grotesquement légaliste de Fluellen dont la science martiale se fonde sur des traités comme celui par exemple de Thomas Digges: An Arithmeticall militarie treatise named Stratioticos réédité en 1590 , où l'on peut reconnaître l'apologie de l'art romain de la guerre ${ }^{21}$ et délibératif dans la scène du camp où le roi se promène anonymement (IV.i.84-222). Si dans les deux premières illustrations de la rhétorique celle-ci joue un rôle plus ornemental qu'heuristique, dans cette scène elle permet d'introduire la problématique de l'Universel et de l'Individuel sous la forme d'une dialectique de la «causa» et du simple soldat. La délibération part d'une double argumentation. Le roi est innocent de la mort de ses soldats: «the king is not bound to answer the particular endings of his soldiers» (1.151-2). Le roi est innocent de leur damnation s'ils meurent en état de péché mortel: «Every subject's duty is the king's, but every subject's soul is his own» (1.171-2). Ce qui semble chez le roi un rigorisme théologique insoutenable est en fait la garantie de la liberté de l'individu par rapport à la cause à laquelle il prête sa substance. À la différence des soldats de $I I$ Henry $I V$, ceux-ci ont des noms propres et non des noms communs déguisés en noms propres. Ce ne sont pas Mouldy, Shadow, Wart, Feeble and Bullcalf, mais John Bates, Alexander Court et Michael Williams. La cause est une chose et eux-mêmes, qui se battent en son nom, en sont une autre. Ils entretiennent avec la cause la même relation que le mot avec la chose.

La question théologique du salut éternel du soldat était selon Ernst Kantorowicz, dans un article intitulé «Pro Patria Mori in medieval thought», un thème de réflexion médiévale ${ }^{22}$. Kantorowicz montre que cette question s'est vue renaître dans une controverse qui oppo- 
sait pendant la guerre de 1914 le cardinal Mercier au cardinal Billot. Le cardinal Mercier soutenait que «la mort chrétiennement acceptée assure au soldat la salut de son âme, tandis que le cardinal Billot rétorque: «Dire que le seul fait de mourir volontairement pour la juste cause de la patrie suffit pour assurer le salut, signifie que l'on substitue la patrie à Dieu, que l'on oublie ce qu'est Dieu, ce qu'est le péché, ce qu'est le pardon divin ${ }^{23}$. Le roi Henry est de cet avis protégeant ainsi ses soldats de la tentation d'assimiler une cause défendue par Dieu et Dieu luimême. Chaque soldat garde son individualité, sa responsabilité devant le péché et c'est un moyen de désacraliser la guerre, lui enlevant le pouvoir divin de blanchir les péchés. Henry se défend ainsi de l'idée du sacrifice des individus à la cause qui sera la cancer de tant d'idéologies bellicistes et totalitaires. Ils ne se sacrifient pas car «they purpose not their death when they purpose their services» (1. 153-4).

Le chœur du début de l'acte IV parle de la métamorphose des Anglais se considérant d'abord comme des sacrifiés puis sous l'influence d'Henry retrouvant leur individualité. «The poor condemned English/like sacrifices» (1.23-4) deviennent plus tard dans la nuit des individus:

Every wretch pining and pale before Beholding him, plucks comfort from his looks A largess universal, like the sun His liberal eye doth give to every one (1.41-44)

Le roi privé de nom pour une nuit, devenu individu au même titre que ses soldats, leur conférant leur.individualité est l'inverse du «word of war» absent du combat qu'était Antoine. La lecture de Henry $V$ montre que si l'idée d'une guerre juste défendue par Dieu est shakespearienne, elle s'accompagne de précautions théologiques qui font qu'il est impossible d'en faire une guerre sainte. C'est une cause sainte mais pas une guerre sainte. La guerre est «impious». Kantorowicz rappelle que le pape Urbain II, qui avait proclamé la première croisade en 
1095. «qualifiait la mort du croisé sur le champ de bataille de charité». Au XIII ${ }^{\mathrm{e}}$ siècle "amor patriae» équivaut à «caritas ${ }^{24} »$. Le simple soldat Michael Williams refuse cette sacralisation de la mort du soldat: «I am afeard there are few die well that die in a battle, for how can they charitably dispose of anything when blood is their argument?» (1.137-40) La question demeure sans réponse, mais c'est le rôle de la rhétorique de poser des questions sans réponses.

La loi de la guerre malgré toutes les précautions prises pour en limiter les excès demeure barbare. À l'ordre du roi de tuer les prisonniers français «Give the word through» (IV.vi.37) ne semble s'opposer aucune disputatio. La scène vi se termine avec cette image ternie du roi qui fait tuer ses prisonniers. Mais voilà qu'avec la scène vii, la rhétorique reprend le dessus. Sans transition Fluellen commente le massacre perpétré par les Français : «Kill the poys and the luggage?' Tis expressly against the law of arms» (IV.vii.1-2). De cette référence à la loi martiale, Gower fait les prémisses d'une justification de la mise à mort des prisonniers. L'ordre du roi était justifié, on le comprend a posteriori (IV.vii.7-10). La justification ne nous satisfait pas mais prouve s'il en était besoin que la problématique de la guerre, même dans ses aspects les plus gênants fait l'objet d'un exercice rhétorique constant. La guerre n'est jamais présente sans que le discours ne cherche à la cerner, à la justifier, à l'excuser, à la faire sans cesse passer du côté du langage.

En faisant de la guerre un objet de rhétorique, Shakespeare aurait pu se limiter à juger de l'opportunité de telle ou telle guerre comme Aristote. Mais que cette rhétorique soit fondée sur la dialectique de la cause et du soldat permet d'aboutir à la primauté de l'individu sur l'Universel. Et ceci paradoxalement grâce à l'argument métaphysique du salut de l'âme du soldat, seul garant dans l'argumentation d'une désacralisation de la guerre.

On comprend que légitimer la rhétorique comme discours fondateur de la guerre pourrait avoir comme conséquence de mettre en doute la nécessité même de la 
guerre. Ne serait-ce pas détourner la rhétorique de sa fonction d'auxiliaire de la politique et comme les sophistes lui donner tout pouvoir sur la politique, au point que, comme dit R. Laufer, elle est toute la politique et la politique n'a pas d'existence en dehors d'elle? ${ }^{25}$ Shakespeare va justement chercher à faire rivaliser les mots avec.la force comme s'il se souvenait de la citation d'Aristote: «Il est aussi honteux de ne pas savoir se battre avec les mots que de ne pas savoir se défendre avec son corps ${ }^{26}$. Il ira même plus loin. Dans ses comédies qui sont des comédies parce que le conflit n'a pas pu se déclarer dans les faits il donnera à la rhétorique la fonction de remplacer la violence, ce qui, d'après $\mathrm{R}$. Laufer, est précisément le symptôme de «la confusion sophiste entre la pouvoir et son instrument ${ }^{27}$.

Enrichie par les thèses nominalistes qui font triompher l'individu sur toutes les logiques mortifères et abstraites, la rhétorique des comédies cherche à fonder la possibilité que la violence cède le pas au langage. Dans $A s$ You Like It, Touchstone démontre que le bain de sang peut être évité en tout bien tout honneur. À la rhétorique du duel qui justifie habilement le passage à l'acte dans des manuels tels que The Booke of Honor and Armes de Sir William Segar ${ }^{28}$, Touchstone oppose une argumentation pacifique. Imaginons le cas où quelqu'un vous accuse de mentir, alors il faut émettre le doute, avoir recours à l'irréel:

I knew when seven justices could not take up a quarrel, but when the parties were met themselves, one of them thought but of an «if»: as «If you said so, then I said so». And they shook hands and swore brothers. Your «if» is the only peacemaker. Much virtue in «if». ( $A Y L$, V.iv.95-100)

Ainsi, la fiction vient au secours de la réalité. La rhétorique a vaincu la guerre. Cette découverte illumine le monde des comédies et le stratagème fait échec à la stratégie. 


\section{NOTES}

(1) J.M. Miller, M.H. Prosser, T.W. Benson, ed., Readings in Medieval Rhetoric (Indiana University Press 1973) 3.

(2) Miller, Readings, 80.

(3) Aristote, Rhétorique (I $41359 \mathrm{~b}$ )

(4) Platon, La République (II 375 b-e)

(5) Platon, Les Lois, (VIII 829a) La guerre conséquence de la tyrannie (République IX)

(6) La guerre, conséquence du péché n'est donc pas fatale. Il faut tout de même se préparer à son éventualité (Les Lois VIII) Dans La Politique d'Aristote, celui-ci reproche à Platon de ne pas donner une place aux soldats dans le livre II de la République (Livre IV, ch.4). On peut parler d'un "pacifisme» platonicien: S. Grésillon, «Platon le Pacifique», La Guerre, dir. de la publ., S. Goyard-Fabre, Cahiers de philosophie politique et juridique (Centre de Publications de l'Université de Caen, 10, 1986) 51-61.

(7) St Augustin, La Cité de Dieu (I, 19)

(8) Erasme, Colloques, Livre I, («Les Introuvables», Éditions d'Aujourd'hui 1983) 179-191.

(9) Platon, Le Politique 308a.

(10) Platon, Le Politique 307e.

(11) voir note 6

(12) Aristote, La Politique, (Livre I chap. 8)

(13) A. Koyré, "Aristotélisme et Platonisme», Études d'histoire de la pensée scientifique (Gallimard, Paris 1973) 33.

(14) in La Guerre, 77-85. Voir note 6.

(15) Michel Meyer, ed. De la Métaphysique à la Rhétorique (Éditions de l'Université de Bruxelles 1986) 189-203.

(16) J. Quillet, «Quelques aspects de la pensée de la guerre au Moyen-Age», La Guerre, 77.

(17) M. Jones-Davies, "Paroles intertextuelles: lecture intertextuelle de Parolles», J. Fuzier et F. Laroque ed. All's Well that Ends Well, Actes du Colloque, Collection Astrea no 1 (Publications de l'Université Paul Valéry 1986) 73-4.

(18) Aristote, La Politique (Livre I chap 8)

(19) M. Meyer «Y a-t-il une modernité rhétorique?», M. Meyer, ed. De la Métaphysique à la Rhétorique, 10.

(20) J. Quillet, «Quelques aspects de la pensée de la guerre au Moyen-Age», La guerre, 77.

(21) W. Shakespearre Henry $V$, ed., A.R. Humphreys (The New Penguin, 1968)191 note de l'acte III ii 70-71.

(22) P. Legendre ed. Ernst Kantorowicz, Mourir pour la Patrie et autres textes, (PUF 1984) 107-141.

(23) E. Kantorowicz, «Mourir pour la patrie (Pro patria Mori) dans la pensée politique médiévale» voir note $22: 107,109$.

(24) E. Kantorowicz, "Mourir pour la patrie...», 133.

(25) R. Laufer, «Rhétorique et Politique», voir note 15: De la Métaphysique à la Rhétorique, 191.

(26) Aristote, Rhétorique, 1355b. Cité et commenté par R. Laufer, «Rhétorique et Politique», 191.

(27) R. Laufer, «Rhétorique et Politique», 191.

(28) W. Shakespearre, As You Like It, R. Knowles ed., A New Variorum Edition of Shakespeare, (The modern Language Association of America, 1977) 290. (note 2664). 